\section{COMPARATIVE STUDIES ON THE ANTICOAGULANT AND ANTIPROTEASE ACTIVITY OF BOVINE, OVINE AND PORCINE HEPARINS}

Tishya Indran, Mohammad Karam Alsarraj, Jawed Fareed, Jeanine Walenga, Walter Jeske, Debra Hoppensteadt, Omer Iqbal, Mamdouh Bakhos

Department of Pathology, Loyola University, Chicago, Maywood, Illinois, USA

Aim: Demonstrate the biosimilarity of the different heparin species.

Method: Bovine, ovine and porcine heparins were obtained with the working concentration of $1 \mathrm{mg} / \mathrm{mL}$. USP standard heparin was used to reference the potency of each of the pooled solutions. Normal human plasma pool was supplemented with each of the Heparin at concentration of $0-1 \mu \mathrm{g} / \mathrm{mL}$. The aPTT, thrombin time, anti-Xa and anti-IIa were used to measure the biological actions.

Results: All the agents produced a concentration dependent increase in aPTT. Porcine heparin produced stronger anticoagulant activity. The USP cross reference activity was $210 \mathrm{U} / \mathrm{mg}$ for porcine, $184 \mathrm{U} / \mathrm{mg}$ for ovine and $104 \mathrm{U} / \mathrm{mg}$ for bovine preparation. In the anti-Xa assay, porcine heparin exhibited a potency of $215 \mathrm{U} / \mathrm{mg}$, ovine $184 \mathrm{U} / \mathrm{mg}$ and bovine $120 \mathrm{U} / \mathrm{mg}$. For the anti-IIa assay, porcine heparin exhibited the highest potency of $160 \mu / \mathrm{mg}$. In the thrombin time assay, porcine heparin produced the strongest anticoagulation activity whereas the bovine and ovine showed comparable results.

Discussion: Heparins isolated from bovine and ovine sources show comparable anticoagulation and antiprotease activities to the porcine product. The potency of ovine and bovine heparin can be adjusted using various assays to exhibit comparable effects to porcine heparins. This study suggests that bovine and ovine heparins are potential substitute anticoagulants for the porcine product.

\section{THE PROGNOSTIC IMPACT OF CD7 EXPRESSION OF LEUKAEMIC BLASTS IN DE NOVO INTERMEDIATE CYTOGENETIC RISK ACUTE MYELOID LEUKAEMIA}

Murali Kesavan $^{1,2}$, Hun Chuah ${ }^{2}$, S.Aqif Mukhtar ${ }^{3,4}$, Ashier Leigh Parsons ${ }^{5}$, Kerryn Stoner ${ }^{6}$, Anastazia Keegan ${ }^{2}$ ${ }^{1}$ The University of Western Australia, Crawley, ${ }^{2}$ Department of Haematology Fiona Stanley Hospital, Murdoch, ${ }^{3}$ Centre for Population Health Research, Curtin University, Bentley, ${ }^{4}$ Department of Health Western Australia Country Health Service, Perth, ${ }^{5}$ Department of Molecular Haematology, and ${ }^{6}$ Flow Cytometry PathWest Laboratory, Murdoch, WA, Australia

Aim: To assess the prognostic impact of CD7 expression in intermediate cytogenetic risk acute myeloid leukaemia (AML).

Method: Twenty-five CD7+ cases were identified from 383 consecutive AML presentations from two Western Australia centres with centralised immunophenotyping, cytogenetic studies and molecular testing from 2007 to 2015. Age, gender, white cell count at diagnosis, cytogenetics, molecular studies (FLT3-ITD, FLT3-ITD allele burden and NPM mutation status) were collected. Kaplan-Meier survival analysis was performed by comparison with an age, sex, cytogenetic and molecular matched cohort.
Results: Median age of diagnosis was 55 years. Of the 12 de novo intermediate risk cases; $17 \%(2 / 12)$ were FLT3+/NPM(allele burden <0.5), $8 \%(1 / 12)$ were FLT3+/NPM+ (allele burden $>0.5$ ) and these patients had the shortest mean survival of 300 days. The remaining 58\% (7/12) were FLT3-/NPM- with a $57 \%$ mortality rate (4/7). The CD7+ de novo cohort had a reduced mean survival time of 669 days compared to 1052 days for the CD7- cohort $(p=0.214)$. Survival curve analysis demonstrated improved overall survival for the CD7+ cohort, however was not statistically significant $(p=0.204)$.

Discussion: Despite the reduced survival observed in the CD7+ AML cohort, statistical significance was not demonstrated. Further studies are required to assess the prognostic effect of CD7 expression in AML.

\section{SHORT HAIRPIN RNA SILENCING OF INTERLEUKIN-6 IN HUMAN BONE MARROW-DERIVED MESENCHYMAL STROMAL CELLS INHIBITS MULTIPLE MYELOMA CELL GROWTH}

Hoon Koon Teoh ${ }^{1,2}$, Pei Pei Chong ${ }^{2}$, Maha Abdullah ${ }^{2}$, Zamberi Sekawi ${ }^{2}$, Geok Chin Tan ${ }^{3}$, Chooi Fun Leong ${ }^{3}$, Soon Keng Cheong ${ }^{4}$

${ }^{1}$ PPUKM-MAKNA Cancer Center, Universiti Kebangsaan Malaysia Medical Centre, Kuala Lumpur, ${ }^{2}$ Faculty of Medicine and Health Sciences, Universiti Putra Malaysia, Selangor,

${ }^{3}$ Faculty of Medicine, Universiti Kebangsaan Malaysia, Kuala Lumpur, and ${ }^{4}$ Faculty of Medicine and Health Sciences, Universiti Tunku Abdul Rahman, Selangor, Malaysia

Mesenchymal stromal cells (MSC) from bone marrow stroma produced high concentrations of interleukin-6 (IL-6) that promoted multiple myeloma growth. More effective methods are needed to disrupt the favourable microenvironment in the bone marrow stroma as earlier trials with IL-6 monoclonal antibody therapy failed to demonstrate significant clinical responses. In this study, RNA interference (RNAi)-mediated silencing of IL-6 in MSC and the efficacy on U266 multiple myeloma cell growth inhibition in vitro and in vivo were evaluated for the first time. RNAi-mediated IL-6 silencing in MSC was induced using vector-based adenovirus vector encoding IL-6 shRNA (pAd/ BLOCK-iT/IL-6). IL-6 protein was significantly suppressed 72 hours post $\mathrm{pAd} / \mathrm{BLOCK}-\mathrm{iT} / \mathrm{IL}-6$ transduction without affecting MSC major biological properties. Subsequent in vitro results showed that U266 growth inhibition was achieved when cocultured with IL-6 shRNA transduced MSC. Nude mice coinjected with IL-6 shRNA transduced MSC also showed significant reduction of U266 tumour volume and tumour mitotic index compared to control mice. In conclusion, IL-6 shRNA transduced MSC displayed in vitro and in vivo antitumor efficacy against multiple myeloma cells suggesting the feasibility of using RNAi as an alternative for targeted suppression of IL-6 in MSC to inhibit multiple myeloma cell growth.

\section{CASE REPORT OF BLASTIC PLASMACYTOID DENDRITIC CELL NEOPLASM (BPDCN) A RARE ENTITY}

Ming Sheng Lim, Anoop K. Enjeti, Karla Lemmert ${ }^{1}$ Haematology Department, Calvary Mater Hospital, Waratah, and ${ }^{2}$ Flow Cytometry Laboratory, Pathology North, John Hunter Hospital, New Lambton, NSW, Australia 